WORK IN PROGRESS

\title{
UTERHAP: Immersive virtual reality simulator for postpartum uterine involution using haptic devices
}

\author{
Francisco Cortes, Diego Vázquez. Eusebio Ricárdez
}

Published: 30 November 2021

\begin{abstract}
This work describes a virtual simulator integrating haptic devices with tactile feedback. The simulator allows users to perform the practice for palpation of uterine involution, where the user immerses in a virtual reality that brings closer to a real practice, the objective of this virtual environment is to speed up the learning process, since it provides practical notions to nursing students before having contact with real patients, highlighting that this simulator does not seek to replace real practice but rather to be an auxiliary tool.
\end{abstract}

\section{Keywords:}

Haptic Device; Virtual Reality Headset; Virtual Reality; Academic training; Virtual environment; Simulator; Mesh Deformer; Tactile Feedback.

\section{Introduction}

Uterine involution is a process that consists of the uterus returning to its usual size after childbirth, the evolution of the uterus through palpation is important to find symptoms that the mother may present, also the progress of the uterine inflammation can to give indications in case the placenta has not been completely extracted, this examination is essential during the time that the patient is under observation after delivery. The foregoing is valued by trained personnel, so practice is required, however, there are no simulators for palpation of uterine involution and nursing students must carry out these practices with real patients.

Therefore, it is important to integrate technology that allows nursing students to have tools that help to recreate real environments where these practices are carried out. With virtual reality, the user is immersed in an environment where their senses are intensified by showing a virtual doctor's office through the Oculus Rift (vision) and interacting with virtual objects to reach them and feel their shape with the Touch 3D Stylus (tactile feedback). Another fundamental part is the deformation of the

Cortes, Francisco., Vázquez, Diego., Ricárdez Eusebio.

Escuela Superior de Ingeniería Mecánica y Eléctrica, Unidad Culhuacán

del Instituto Politécnico Nacional

México, México City

fj23pk@gmail.com,diego.vazpez@hotmail.com, eusebior@ieee.org. objects, by touching them, the user can perceive that he is interacting with a soft body (depth) and deduce how much force to apply to the haptic device. This virtual simulator with tactile feedback is a first approach for the students prior to the real practices, it was tested by teachers and the opinions were varied according to their experience, in general, they were interested and saw a future in virtual simulators.

\section{Related Work}

Below is a brief description of projects and research related to uterine involution simulators as well as the use of haptic devices.

The first work is the resectoscopy simulator is described in [1] for uterine resectoscopy in virtual reality, which by means of a mechanical uterus can be inserted the resectoscope and visualize on the computer screen how the examination progresses in the intrauterine environment.

Next in [2] which is a patent for a physical simulator for medical processes in the uterine area, this type of simulator is made of elastomeric material that simulates the postpartum abdominopelvic soft tissue very similar to the texture of the cavernous lining of the uterus. This allows simulating various techniques such as performing surgery, either for reasons unrelated to childbirth or merely obstetric and gynecological.

Later in [3] which the evaluation of the results of a study carried out with health personnel in Ecuador is discussed, where training was implemented for the placement of a device for hemostasis, to reduce uterine bleeding in postpartum patients.

After reviewing the related work, different opportunity areas were identified where virtual simulators can be developed:

In the previous works, the use of haptic devices is not presented, in such a way that the simulators are physical and can be a disadvantage since elastomeric materials might be damaged with the passage of time or improper use.

In simulators for uterine processes there is no specific model for palpation of uterine involution, it can be done, but the characteristics of the uterus are not adequate.

\section{UTERHAP}

According to the advice provided by the head of the Department of Obstetric Nursing Training at Escuela Superior de Enfermería y Obstetricia del Instituto Politécnico Nacional (ESEO - IPN), who mentions that the practice for palpation of uterine involution is carried out in the last semesters of the nursing career in person.

For the practice of palpation, one hand is required to exert force on the uterus and the other only to hold, so it was decided to use only a haptic device. In Figure 1 is shown the form of postpartum uterine palpation. 


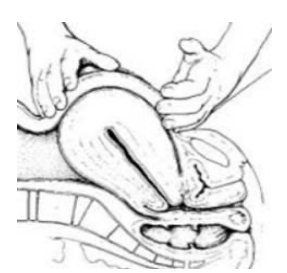

Figure 1. Uterus palpation in puerperium [4].

\subsection{Simulator architecture}

The simulator architecture for palpation of uterine involution is based on the work of [5] with some modifications, as shown in Figure 2, which also uses haptic devices. The arrows in the diagram indicate the communication between the blocks, the user receives the video output through the Oculus and with the haptic device sends and receives tactile feedback. The operation of each block is described below.

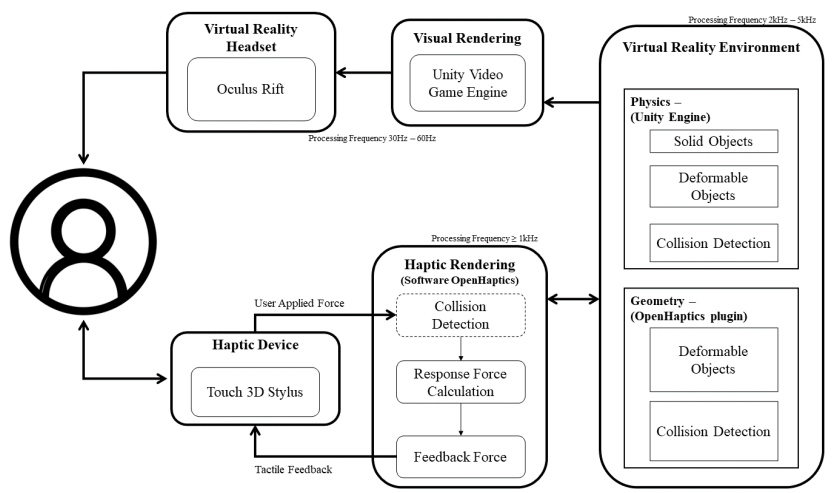

Figure 2. Simulator architecture using operating blocks.

\subsubsection{Virtual Reality Environment (VRE)}

The most important part is in the simulator operation, where the doctor's office and the medical supplies serve as a visual tool for the user. The virtual models take physical characteristics (mass, gravity, collision detection) to interact with them, for this the physical manager of Unity [6] was used. The Unity engine allows calculating collisions between objects, in this case, being a virtual world, it is necessary to identify the position of the objects in that space.

The 3D Systems OpenHaptics plugin obtained from the AssetStore [7] was also used, which allows haptic effects such as static and dynamic friction, damping, stiffness and to pop through virtual objects with previously configured physical characteristics, the objects with these qualities: models uterus (see Figure 3) considering the phases of the puerperium: immediate (first $24 \mathrm{~h}$ ) and mediate (day 2 to 7 ), whereas the days go by the size of the uterus decreases.
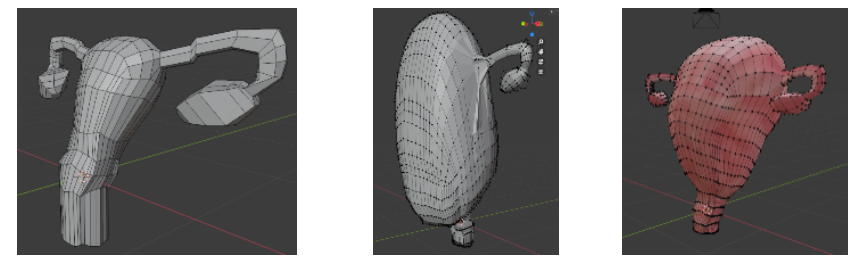

Figure 3. Uterus models: immediate and mediate puerperium.
The quality of mesh deformation is integrated in 3D virtual objects, when two objects collide and at least one has this deformation quality, it will undergo a deformation in its meshes that after a while will return to its original shape, this property is also known as elastic movement, the object with this quality is the abdominopelvic model.

For this block it is necessary to have two linked haptic devices (virtual and physical), when the user moves the physical device on the screen will see the virtual device move, the same happens when the user interacts with virtual objects. For this reason, a hand model was designed as a user avatar (see Figure 4), it is configured as a controller called grabber or handle and limited in a workspace called hapticDevice, which is an invisible cube for the user and only in that space will work haptic properties. These last two settings are from the OpenHaptics plugin.

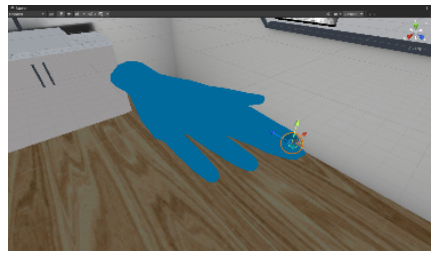

Figure 4. User avatar: a hand model as a haptic device.

\subsubsection{Haptic Rendering}

Although the simulation processes are carried out in the VRE block, the physical communication between the haptic device and the virtual environment is important, for this work the haptic development software [8], this software calculates the real position of the haptic device according the movement and force applied by the user, if there is a collision with virtual objects configured in VRE, tactile feedback (force) is obtained, this block needs processing frequency at least $1 \mathrm{kHz}$.

\subsubsection{Visual Rendering}

The Unity graphics engine is responsible for collecting all the necessary libraries to display the video output on the screen with the help of OpenGL. However, the main output of the simulator is the Oculus Rift, for this the Oculus own software is used that allows communication between the device and the computer, as for Unity, the XR Plugin Management is obtained in the package manager that provide the necessary scripts to configure the virtual reality camera linked to the Oculus. Then the user can choose on which device to view the virtual environment. The processing frequency is between $30 \mathrm{~Hz}$ and $60 \mathrm{~Hz}$.

\subsection{D Models Behavior}

As mentioned, the simulator must be immersive, which is why the abdominopelvic area requires the deformation effect as occurs when touching the real skin, then to add more realism to simulation. So, the abdominopelvic model was designed independent to female body, in this way to add mesh collider characteristic and mesh deformer and deformation is configured as shown in Figure 5.

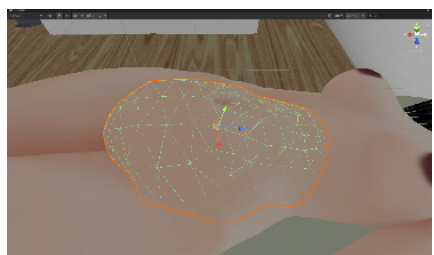

Figure 5. Abdominopelvic model with mesh collider characteristic. 


\subsubsection{Mesh Deformation}

The mesh deformation script was written to calculate the position of 3D model vertices with physics qualities, through mesh filter provided by the Unity physic engine to identify the initial position values which will be used later to return original form. Two forces are required for this script: applied force and the offset force to compensate the force with which the meshes are pushed radially or the inverse force applied with the user avatar in Figure 6 is shown the flow chart to mesh deformation.

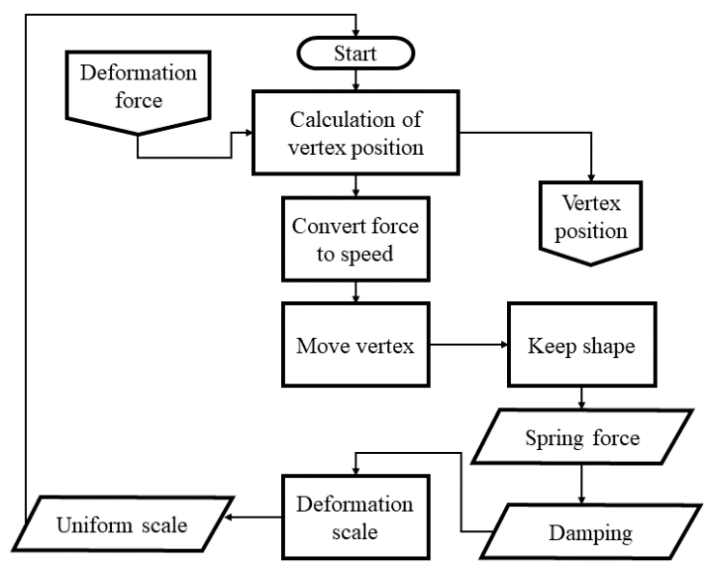

Figure 6. Mesh deformation flow chart.

The vertices are pushed by the applied force; however, it is necessary to identify the speed and direction of that push. The applied force $(F)$ becomes speed $\left(F_{v}\right)$, this is where the inverse square law applies where the original force is divided by one plus the distance squared.

$$
F_{v}=\frac{F}{1+d^{2}}
$$

This guarantees that the force is maximum when the distance is zero $(d)$, so, the greater the distance, the lower the speed.

Regarding the direction, it is enough to normalize the vector of the applied force. Although the vertices are already moving, their position and normals must be recalculated as fast as processing frequency between $2 \mathrm{kHz}$ to $5 \mathrm{kHz}$, this maintains the original shape of the mesh. This is done by applying elastic force or springs to move the vertices in an inverse way but with a different speed from that applied. This speed is calculated by the difference of the initial thrust speed and the product of the displacement, spring force and time. Every time a vertex moves in reverse, the speed decreases.

Now, it is possible to move the vertices and return them to their original position, however, due to the nature of the position calculation, it tends to repeat itself infinitely. To solve this oscillation, the application of damping is used, with which the speed of the displacement is further decreased over time, thus returning the vertices to their original position.

When moving the vertices, it is assumed that the scale of the $3 \mathrm{D}$ object changes, the object might grow or becomes small, to compensate for this action it is first necessary to identify the original scale of the object and then compare it with the scale when the vertices have displaced. Then, the scale is readjusted so as not to lose its uniformity, this is called meshDeformation.

\subsubsection{Mesh Deformer}

To perform the deformation in the abdominopelvic area at the contact point of the user avatar or meshDeformer, the mesh deformer script was written (see Figure 7), it is necessary to obtain the coordinates of that contact point. First, the position of the user avatar is calculated, the Haptic Interaction Point (HIP) was located at the tip of the index and middle fingers, this sphere is considered the origin for the projection of a shock beam and the direction used is the inverted $\mathrm{Y}$ axis. Then, a ray that is not visible to the user comes out of the user avatar, in such a way that when making contact with the area to be deformed, the coordinates of the point where the ray collided with the mesh collider are calculated. At that point the deformation force is applied to move the vertices, an action performed by the mesh deformation script.

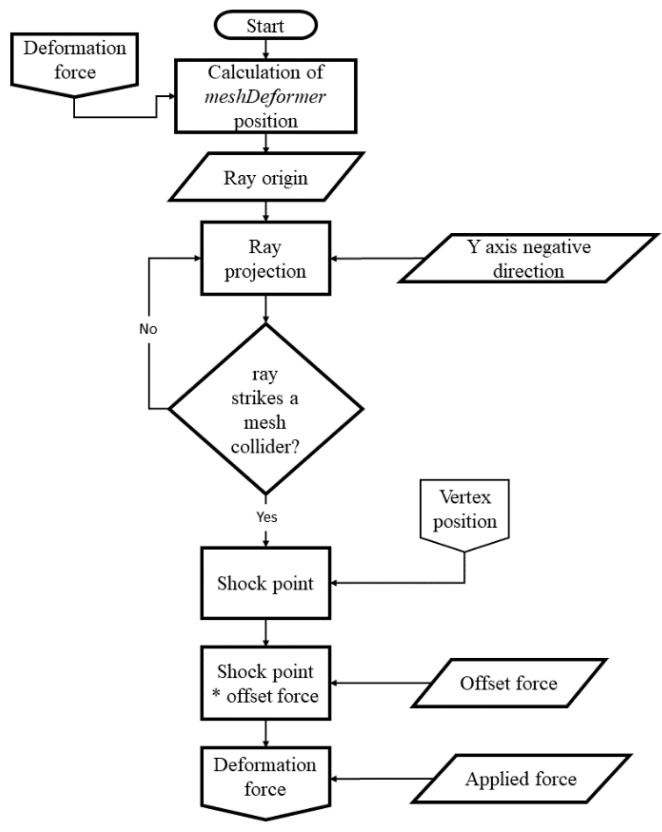

Figure 7. Mesh deformer flow chart.

\section{Preliminary Results}

To carry out the preliminary tests, 5 volunteers attended the presentation of the simulator for palpation of uterine involution. All the participants are nurses and teachers at ESEO - IPN, aged between 33 and 50 years old, experts in uterine palpation during the puerperium. For these tests, the equipment used was a laptop ASUS with intel I7-9750H @ 2.50 GHZ processor, 16 Gb. RAM, Nvidia GeForce RTX 2060 graphic card, 1 HDMI port and 4 USB 3.0 ports. Also, one Oculus Rift headset and the haptic device Touch 3D Stylus were used.

For practice, the user must be seated in front of the computer, put on the Oculus Rift and hold the Touch 3D Stylus with the dominant hand, before starting the simulation (see Figure 8). User was instructed about the actions that could perform within the virtual environment: observe the doctor's office by moving the head, with the haptic device touching the abdominopelvic area and when applying pressure could feel different stiffness depending on the phase of the puerperium.

The default setting selects the uterus in the immediate puerperium, with the uterus being the largest. Then the user can select another uterus from those available. At the end of the practice, a brief questionnaire was made to the participants where the answer could be agree, indifferent or disagree. Although the participants shared their experience through a comparison of real practice with this simulation. The results shown in the Figure 9 were obtained. 

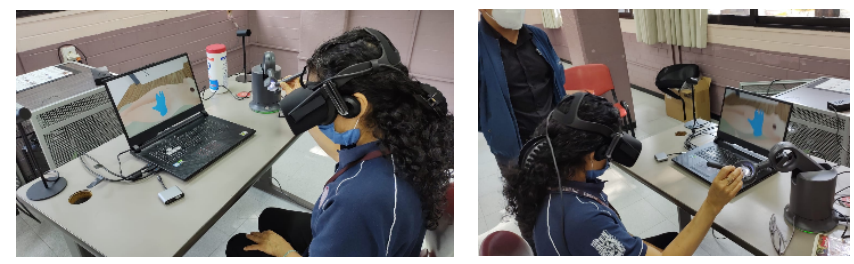

Figure 8. User performing the uterine palpation in UTERHAP.

It is observed that $95 \%$ agree that the feedback force is similar to a real practice. $80 \%$ agree that when interacting with objects their shape and texture can be felt. $70 \%$ agree with the perception of depth when touching the skin and seeing how it deforms. 50\% were able to identify the postpartum phase by palpating the uterus with the haptic device. And $100 \%$ of the users experienced the immersion of being inside a virtual medical environment.

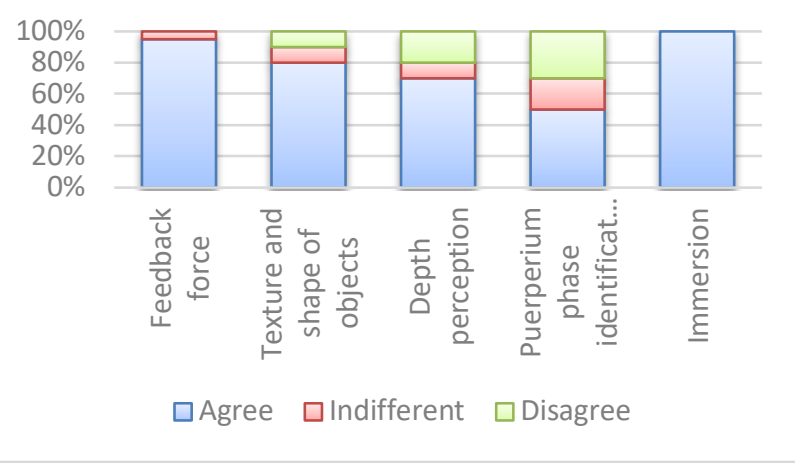

Figure 9. Questionnaire results made to the participants at the end of the tests.

\section{Conclusions and Future Work}

In both medicine and engineering, it is important to have tools that help students acquire the necessary skills. In this case, the use of haptic devices with tactile feedback and a virtual reality viewer immerses the user in a virtual environment to perform virtual practices that are reliable to what would be done in a real practice.

So, the contribution of this work is to simulate the palpation of uterine involution using virtual models with tactile properties that can be felt with a haptic device and the recreation of a virtual medical environment with elements that allow the user to immerse themselves in that with a headset. At the same time, the practitioner is made aware of how this practice should be carried out without disturbing or damaging real patients. Even perform this practice at any time and as many times as necessary regardless of the number of people who are observing the uterine exploration process. The work developed stands out because there was no simulator of this type, however, areas of opportunity are identified that can allow improvements to this work.

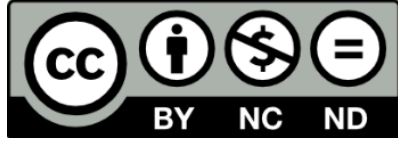

Graphics improvement. To optimize the ratio of graphics quality and simulator performance.

Improved touch parameters. Perform more tests with nurses to further fine-tune the haptic characteristics.

Integration of any haptic device. The simulator supports 3D Systems OpenHaptics compatible devices but others could be added.

\section{Acknowledgements}

Thanks to Dr. José Velázquez López for their support and advice for the technological development of this project. To M. in D. Flor de María Cruz García from ESEO for her advice on the investigation of medical aspects. Also, to ESEO staff who gave their feedback when using the simulator during the tests.

\section{References}

[1] M. M. Gordon and B. D. Paul, "Virtual Reality Uterine Resectoscopic Simulator: Face and Construct Validation and Comparative Evaluation in an Educational Environment," Society of Laparoscopic \& Robotic Surgeons, vol. 2, pp. 142146, June 2011.

[2] S. Vivienne and M. Morag, "Simulator for training medical personnel to perform uterine procedures". Patent 10235903, March 2019.

[3] P. D. Avilés Terneux, "Evaluación de los resultados de aprendizaje del uso del balón de taponamiento intrauterino "Sayeba" en el control de la hemorragia postparto con simuladores de baja fidelidad en el personal de salud pública de la provincia de Carchi, de septiembre a octubre de 2017," PUCE, Quito, 2018.

[4] A. A. Díaz, "Complicaciones Puerperales: Atonía uterina," Universidad de Valladolid. Facultad de Enfermería de Valladolid., Valladolid, 2015.

[5] E. Ricardez, J. Noguez, L. Neri, D. Escobar-Castillejos and L. Muñoz, "SutureHap: Use of a physics engine to enable force feedback generation on deformable surfaces simulations," International Journal of Advanced Robotic Systems, vol. 15, no. 1, p. 83, January 2018.

[6] Unity Technologies, "Unity," 2021. [Online]. Available: https://unity.com/. [Accessed 2021].

[7] Unity Technologies, "3D Systems Openhaptics ${ }^{\circledR}$ Unity Plugin," Unity Asset Store, 2019. [Online]. Available: https://assetstore.unity.com/packages/tools/integration/3dsystems-openhaptics-unity-plugin-134024. [Accessed 2021].

[8] 3D Systems, "OpenHaptics, Haptic developer software," [Online]. Available: https://www.3dsystems.com/hapticsdevices/openhaptics. [Accessed 2021].

(C) 2021 by the authors. This work is licensed under the Creative Commons AttributionNonCommercial-NoDerivatives 4.0 International License. To view a copy of this license, visit http://creativecommons.org/licenses/by-nc-nd/4.0/ or send a letter to Creative Commons, PO Box 1866, Mountain View, CA 94042, USA. 